\title{
Nonlinear time-series forecasts for decision support: short-term demand for ICU beds in Santiago, Chile during the 2021 COVID-19 pandemic
}

Bernardo F. Quiroga ( $\sim$ bfquirog@uc.cl)

Pontificia Universidad Católica de Chile https://orcid.org/0000-0002-0465-0460

Cristián Vásquez

Pontificia Universidad Católica de Chile

María Ignacia Vicuña

Pontificia Universidad Católica de Chile

\section{Method Article}

Keywords: COVID-19, Modeling biological systems, Infectious disease dynamics, Time series forecasting

Posted Date: September 21st, 2021

DOl: https://doi.org/10.21203/rs.3.rs-922400/v1

License: (c) (i) This work is licensed under a Creative Commons Attribution 4.0 International License.

Read Full License

Version of Record: A version of this preprint was published at International Transactions in Operational Research on October 20th, 2022. See the published version at https://doi.org/10.1111/itor.13222. 
Preprint (2021) 1-19

\title{
Nonlinear time-series forecasts for decision support: short-term demand for ICU beds in Santiago, Chile during the 2021 COVID-19 pandemic
}

\author{
Bernardo F. Quiroga ${ }^{\mathrm{a}, *}$, Cristián Vásquez ${ }^{\mathrm{a}}$ and María Ignacia Vicuña ${ }^{\mathrm{a}}$ \\ ${ }^{a}$ School of Management, Pontificia Universidad Católica de Chile, Av. Vicuña Mackenna 4860, Macul, Santiago RM 7820436 , \\ Chile \\ E-mail:bfquirog@uc.cl[Quiroga]; ctvasque@uc.cl[Vásquez]; mivicuna@uc.cl [Vicuña]
}

Received 13 September 2021

\begin{abstract}
In Chile, due to the explosive increase of new COVID-19 cases during the first part of 2021, the ability of health services to accommodate new incoming cases was jeopardized. It has become necessary to be able to manage intensive care unit (ICU) capacity, and for this purpose, monitoring both the evolution of new cases and the demand of ICU beds, has become urgent. This paper presents short-term forecast models for the number of new cases and the number of COVID-19 patients admitted to ICUs in the Metropolitan Region in Chile.
\end{abstract}

Keywords: COVID-19; Modeling biological systems; Infectious disease dynamics; Time series forecasting

\section{Introduction}

The pathogen SARS-Cov-2 has caused the infection called Coronavirus disease 2019 (COVID-19) (Andersen et al., 2020), spreading worldwide in a few months since its identification in December 2019. Causal theories of accidental release from a lab and zoonotic spillover both remain viable (Bloom et al., 2021), and it is an ongoing pandemic at the time of writing.

This research aims to provide a framework for decision support for policy makers who need to know in advance how much intensive care unit (ICU) bed capacity needs to be provided to fulfill demand of COVID-19-related ICU hospitalizations via time-series-based forecasts of both number of new COVID19 cases and corresponding ICU bed utilization. We illustrate our proposed methodology with the case of the Metropolitan Region of Santiago, the capitol city of Chile, with data collected during the second wave of the pandemic, the initial four months of 2021.

\subsection{Context}

By late July 2020, with declining rates of new cases, Chile had achieved a partial control of the growth rates of COVID-19. However, with the policy of indiscriminate issuance of Summer Vacation Permits by the Chilean Ministry of Health $(\mathrm{CMoH})$, as announced on December 30th 2020 (Chilean Ministry of

${ }^{*}$ Corresponding author: Bernardo F. Quiroga (e-mail: bfquirog@uc.cl) 
Health, 2020) and continuing until late February 2021, the spread of the virus during the first quarter of 2021 became more pronounced than during its first wave in 2020. Subsequent lockdown policies have been announced in March and April 2021, but have not been effectively enforced by the authorities. Moreover, as Joshi and Musalem (2021) found, lockdown policies have seen their desired effect (in reduced mobility) sliced in half after a month.

With the ability of health services to accommodate new incoming cases in jeopardy, it has become an urgent necessity to be able to manage ICU capacity, and for this purpose, to obtain accurate forecasts of COVID-19 demand for ICU beds. With this reality in mind, and understanding the importance of modeling techniques in order to improve healthcare delivery and informatics (Gupta and Sharda, 2013), we have set ourselves to forecast and model the short-term demand of ICU beds in Santiago, Chile.

\subsection{Literature review and modeling approaches}

A natural inclination for modelers attempting to predict demand for COVID-19 ICU beds, is to use variations of the so-called Susceptible-Infectious-Recovered (SIR) model (Keeling and Danon, 2009; Papo et al., 2020). However, as different authors have remarked (Manca et al., 2020; Gitto et al., 2021), although SIR-type models are good for running parametric predictive scenarios, they are based on many assumptions and hypotheses that are quite sensitive to the selection of proper values of the adaptive parameters and functional description, which might not be as appropriate when real-time data are incomplete.

Similarly, compartment models to study ICU beds utilization (see Goic et al. (2021) as an example) require accurate information on the count of infectious individuals who show COVID-19 symptoms, critically ill people who need an ICU bed, and discharged patients from the ICU, and if such data is not available, an alternative approach becomes necessary.

A key input variable for the short-term forecast of demand of ICU COVID-19 beds is the count of new infected cases, making necessary to also model those cases in order to use them for the demand forecasts. Some authors (e.g., Meo et al. (2020)) have produced forecasts using standard linear regression models, which are less than ideal given the nature of discrete count data.

We have, thus, decided to use phenomenological models to forecast the count of new infected cases, and with that input, obtain ICU bed demand forecasts. Phenomenological models (e.g., Chowell (2017); Chowell et al. (2019); Wang et al. (2012); Roosa et al. (2020b); Chowell et al. (2016), among others) have been previously applied to various infectious disease outbreaks including other respiratory illnesses. This family of models can produce good forecasts using small samples, even in the absence of hypotheses about the population, the rate of diffusion, and the upper limit of the infection curve (Ma et al., 2014; Gitto et al., 2021). Real-time short-term forecasts generated from such models are useful to guide the allocation of resources that are critical to bring the epidemic under control. For instance, Maier and Brockmann (2020) fitted a sub-exponential growth curve for China, whereas Remuzzi and Remuzzi (2020) used exponential growth models to predict the early propagation of COVID-19 in Italy. Sadly, exponential growth models are unrealistic in scenarios where additional information is available. Several authors (e.g., Aviv-Sharon and Aharoni (2020); Vicuña et al. (2021); Roosa et al. (2020a); TorrealbaRodríguez et al. (2020); Zreiq et al. (2020); Chen et al. (2020), among others) propose instead to use generalized variations of the Richards/logistic curve model (Richards, 1959) to generate forecasts of COVID-19 cases in several countries. Gitto et al. (2021) were the first to propose the use of Harvey's model (Harvey, 1984) to forecast the diffusion of an epidemic precisely in the context of bed occupancy in Italy during the first wave of COVID-19. Moreau (2020) used Weibull curves to estimate new cases of COVID-19 in Brazil.

To conduct our own forecasts, we model the evolution of new cases using three phenomenological curves (Richards/logistic, Harvey, Weibull), with different distributional assumptions on the error term. 
In section 2, we describe our modeling strategy in detail, in section 3 we present and discuss our empirical results, and in section 4 we conclude.

\section{Methods}

\subsection{Data}

Data used in this work has been retrieved from the epidemiological reports from the $\mathrm{CMoH}$. These epidemiological reports are updated by the independent expert panel working with the $\mathrm{CMoH}$, and are updated regularly to adjust for errors and misreports. These most accurate counts are collected on the Chilean Ministry of Science (CMoSc) website at https://www.minciencia.gob.cl/covid19. These are the data that other published studies, such as Vicuña et al. (2021); Canals et al. (2020); Tariq et al. (2021), have utilized for the Chilean case.

The dataset includes the total count of confirmed cases in the Metropolitan Region of Santiago, according to Polymerase chain reaction test (PCR) prognosis notification date (as registered by the physician on the $\mathrm{CMoH}$ surveillance system), as well as the daily demand of ICU beds for COVID-19 patients. Fortunately, since the COVID-19 response system has integrated the bed availability network, observed demand is not censored: Every time that capacity has approached $100 \%$ occupancy, the system has been able to open new slots by reconverting beds to ICU use as needed.

Since our study is based on secondary data from the CMoH's official daily public reports as published by the $\mathrm{CMoSc}$, it did not require approval from an Ethics Committee.

\subsection{Modeling strategy}

Our modeling strategy follows a two-stage model to forecast the number of COVID-19 ICU beds demanded per day. For the first stage, we forecast the number of new COVID cases, which is a crucial input for the second stage, particularly for our out-of-sample, short-term forecasts of demand of ICU COVID-19 beds. In the second stage, we forecast the number of beds using the lagged measure of the number of cases the previous week as well as the demand of ICU beds the day before.

\subsubsection{First stage: new cases}

To model the new cases, we use phenomenological models. Many functional forms have been used in epidemic forecasting research. We focus on three popular nonlinear specifications: Richards/logistic (Richards, 1959), Weibull (Weibull, 1951), and Harvey (Harvey, 1984). In terms of notation, let $t$ be a daily time index; $X_{t}$ the observed number of new cases in Santiago on day $t ; \Lambda_{t}$ the cumulative rate of cases on day $t$; and $\lambda_{t}=d \Lambda_{t} / d t$ the corresponding daily arrival rate.

The logistic curve is given by equation (1), whereas the Weibull curve for accumulated cases corresponds to equation (2):

$$
\begin{aligned}
\Lambda_{t}^{(L)} & =\frac{K}{\left[1+\exp \left(-r\left(t-t_{m}\right)\right)\right]}, \\
\Lambda_{t}^{(W)} & =K\left[1-\exp \left[-\exp \left(r \ln (t)-\ln \left(t_{m}\right)\right)\right] .\right.
\end{aligned}
$$

Given that the epidemiological curves in (1) and (2) are used to model the cumulative number of cases, in order to make a daily forecast of new cases, we use for estimation the first derivatives, $\lambda_{t}$, yielding a measure of the daily amount of new cases: 


$$
\begin{aligned}
\lambda_{t}^{(L)} & =r K \frac{\exp \left(-r\left(t-t_{m}\right)\right)}{\left[1+\exp \left(-r\left(t-t_{m}\right)\right)\right]^{2}} \\
\lambda_{t}^{(W)} & =\frac{K r}{t_{m}}\left(t / t_{m}\right)^{r-1} \exp \left[-\exp \left(r\left(\ln (t)-\ln \left(t_{m}\right)\right)\right]\right.
\end{aligned}
$$

Harvey (1984)'s curve, displayed as equation (5), provides a direct forecast the count of new cases during a pandemic. It includes a deterministic time trend and an auto-regressive term to account for the previously accumulated cases:

$$
\ln \left(\lambda_{t}^{(H)}\right)=\delta+r t+\rho \ln \left(\sum_{i=t_{0}}^{t-1} X_{i}\right) .
$$

In order to be able to estimate these curves, we have rewritten the expressions for $\lambda_{t}$ for new cases as function of parameters $\vartheta_{j}$ to be estimated, according to expressions (6), (7) and (8).

$$
\begin{aligned}
\lambda_{t}^{(L)} & =\vartheta_{1} \frac{\exp \left(-\vartheta_{2}\left(t-\vartheta_{3}\right)\right)}{\left[1+\exp \left(-\vartheta_{2}\left(t-\vartheta_{3}\right)\right)\right]^{2}}, \\
\lambda_{t}^{(W)} & =\vartheta_{1}\left(\frac{t}{\vartheta_{3}}\right)^{\vartheta_{2}-1} \exp \left[-\exp \left(\vartheta_{2}\left(\ln (t)-\ln \left(\vartheta_{3}\right)\right)\right]\right. \\
\ln \left(\lambda_{t}^{(H)}\right) & =\vartheta_{1}+\vartheta_{2} t+\vartheta_{3} \ln \left(\sum_{i=1}^{t-1} X_{i}\right) .
\end{aligned}
$$

We thus use these specifications for the rate of new cases as parametric inputs for two probability models: (Quasi-)Poisson and Gaussian. We use Gaussian for benchmarking purposes, keeping in mind that our data constitute discrete (and not continuous) variables.

Since we denoted $\left\{X_{t}\right\}$ as the number of confirmed COVID-19 cases at time $t$, let now $\mathcal{X}_{t-1} \equiv$ $\left\{X_{t-1}, X_{t-2}, \ldots\right\}$ be a collection of all past realizations of $X_{t}$. Then, the Poisson model, used traditionally to analyze count data (see Agosto and Giudici (2020); Barroilhet et al. (2021); Biswas et al. (2020) as examples) has a probability function given by:

$$
\operatorname{Pr}\left(X_{t}=x \mid \mathcal{X}_{t-1}\right)=\frac{\lambda_{t}^{x} \exp \left(-\lambda_{t}\right)}{x !}, \quad x=0,1,2, \ldots
$$

The key handicap of the traditional Poisson model, in this context, comes from its assumption that the mean of the daily number of cases is identical to its variance, i.e., $\mathrm{E}\left(X_{t} \mid \mathcal{X}_{t-1}\right)=\operatorname{Var}\left(X_{t} \mid \mathcal{X}_{t-1}\right)=\lambda_{t}$. The Quasi-Poisson model (as used by Biswas et al. (2020) and Vicuña et al. (2021) among others), instead, offers a generalization allowing for an overdispersion factor $\phi$ :

$$
\begin{array}{r}
\mathrm{E}\left(X_{t} \mid \mathcal{X}_{t-1}\right)=\lambda_{t} \\
\operatorname{Var}\left(X_{t} \mid \mathcal{X}_{t-1}\right)=\phi \lambda_{t}
\end{array}
$$

As mentioned before, for benchmark/comparison purposes, we also fit a Gaussian (Normal) model, with mean $\mathrm{E}\left(X_{t} \mid \mathcal{X}_{t-1}\right)=\lambda_{t}$ and variance $\operatorname{Var}\left(X_{t} \mid \mathcal{X}_{t-1}\right)=\sigma_{t}^{2}$. Its density is given by:

$$
f_{X_{t}}\left(x \mid \mathcal{X}_{t-1}\right)=\frac{1}{\sigma_{t} \sqrt{2 \pi}} \exp \left(-\frac{1}{2}\left[\frac{x-\lambda_{t}}{\sigma_{t}}\right]^{2}\right)
$$


In this first stage, our objective is to obtain the rate of new COVID-19 cases via the conditional means $\lambda_{t}$ specified through equations (6), (7), and (8), as well as other covariates. Specifically, (i) the variable WeekDay $_{j, t}$ is an indicator variable for each day of the week, capturing the effect of the reduced capacity in the release of PCR test results during weekends (see Vicuña et al. (2021); Nakagawa and Kanatani (2021) as examples); (ii) the variable $I_{t}$ is an indicator capturing a full lockdown policy intervention in Santiago (in our sample, between March 27th and April 28th 2021); (iii) the parameter $\delta_{0}$ is an offset (intercept) value which captures the fact that the sample under analysis didn't start from zero cases. Therefore, the conditional mean used for estimation is given by equation (9):

$$
\lambda_{t}=\left(\delta_{0}+\lambda_{t}^{(\ell)}+\exp \left(\psi I_{t}\right)\right) \exp \left(\sum_{j=1}^{7} \beta_{j} \text { WeekDay }_{j, t}\right), \ell \in\{L, W, H\}
$$

For the Quasi-Poisson case, the parameters to be estimated for the proposed curves (all collected in a vector called $\theta$ ) are estimated by maximizing the quasi-likelihood function (given by expression (10)):

$$
\widehat{\theta}=\arg \max _{\theta} \sum_{t=1}^{n}\left[\frac{1}{\phi}\left(y_{t} \ln \left(\lambda_{t}\right)-\lambda_{t}\right)+\kappa\left(y_{t}, \phi\right)\right],
$$

where $\kappa(y, \phi)=\phi^{-1}[-y \ln (y)+y]$.

Similarly, for the Gaussian case, the parameters $\theta$ are estimated by maximizing a log-likelihood function as in expression (11):

$$
\widehat{\theta}=\arg \max _{\theta} \sum_{t=1}^{n} \ln f_{X_{t}}\left(x \mid \mathcal{X}_{t-1}\right),
$$

These results allow us to obtain an estimated value for the expected rate of arrival of new cases in day $t$ in Santiago, $\lambda_{t}$, which is the key input for our second stage model to estimate the demand for ICU beds.

\subsubsection{Second stage: demand for COVID-19 ICU beds}

To model the short-term daily demand for COVID-19 ICU beds in Santiago, we propose to fit generalized Poisson and Quasi-Poisson time series models. The variables chosen as predictors are (i) the (lagged) count of new COVID-19 diagnosed cases, and (ii) an auto-regressive term accounting for the utilization of ICU beds by COVID-19 patients in the immediately preceding days.

It is important to remember that, as mentioned earlier, in the case of Santiago (and Chile in general), utilization of ICU beds by COVID-19 patients equals demand, since there has been no demand censoring effect: thus far, whenever utilization has approached $100 \%$ of available ICU beds, the integrated health system for COVID-19 has ensured that new capacity has been created for incoming COVID-19 patients needing ICU care. Therefore, the terms utilization of and demand for ICU beds by COVID-19 patients, can be used indistinctly in this case.

Denote as $\left\{Y_{t}\right\}$ the stock of utilized ICU beds for COVID-19 cases in Santiago on day $t$. As before, let $\left\{X_{t}\right\}$ correspond to the count of new COVID-19 cases diagnosed on day $t$. Consider $\mathcal{F}_{t-1}:=$ $\left\{Y_{t-1}, X_{t-1}, Y_{t-2}, X_{t-2}, \ldots\right\}$, a collection of all realizations of $\left\{X_{t}\right\}$ and $\left\{Y_{t}\right\}$ from the beginning of the sample until $t-1$. Then, the Poisson time series assumes that $Y_{t}$ conditional on $\mathcal{F}_{t-1}$ is well-described by the following probability function:

$$
\operatorname{Pr}\left(Y_{t}=y \mid \mathcal{F}_{t-1}\right)=\frac{\mu_{t}^{y} \exp \left(-\mu_{t}\right)}{y !}, \quad y=0,1,2, \ldots
$$


In this model, $\mathrm{E}\left(Y_{t} \mid \mathcal{F}_{t-1}\right)=\operatorname{Var}\left(Y_{t} \mid \mathcal{F}_{t-1}\right)=\mu_{t}>0$. Here, the rate $\mu_{t}=g\left(\mathcal{F}_{t-1}, \alpha\right)$ is a function of the covariates associated to the response variable $Y_{t}$ and parameters that need to be estimated.

The proposed model for the case of Santiago considers the following specification for the mean demand $\mu_{t}$ :

$$
\mu_{t}=\alpha_{0}+\sum_{k=1}^{p} \alpha_{k} Y_{t-k}+\sum_{l=1}^{q} \beta_{k} \mu_{t-k}+\eta_{1} X_{1, t}+\eta_{2} X_{2, t}
$$

where $Y_{t}$ is the utilization of COVID-19 ICU beds on day $t ; \mu_{t}$ is the mean (demand) rate of the process on day $t$; covariate $X_{1, t} \equiv \ln \left(\sum_{i=1}^{t-1} Y_{i}\right)$ corresponds to a Harvey-like (Harvey, 1984) growth curve component, capturing the similarities between the use of beds and the count of new cases; and covariate $X_{2, t} \equiv \bar{X}_{t-\tau}=\frac{1}{7} \sum_{i=t-(7-\tau-1)}^{t-\tau} X_{i}$ is a 7-day moving average of the daily count of new cases with an out-of-phase lag of $\tau$ days, capturing the effect of a full week of new infected cases on the future utilization of beds $\tau$ days ahead.

To estimate the unknown parameters, we use the following log-likelihood function given by (12):

$$
\mathcal{L}(\boldsymbol{\omega})=\sum_{t=1}^{n} \ln p_{t}\left(y_{t} ; \boldsymbol{\omega}\right)=\sum_{t=1}^{n}\left(y_{t} \ln \left(\mu_{t}(\boldsymbol{\omega})\right)-\mu_{t}(\boldsymbol{\omega})\right),
$$

where $\boldsymbol{\omega}=\left(\alpha_{0}, \alpha_{1}, \ldots, \alpha_{p}, \beta_{1}, \ldots, \beta_{q}, \eta_{1}, \eta_{2}\right)$, and $p_{t}\left(y_{t} ; \boldsymbol{\omega}\right)=\operatorname{Pr}\left(Y_{t}=y \mid \mathcal{F}_{t-1}\right)$ is the Poisson probability function. Thus, the estimator of the unknown parameters is obtained by maximizing the following program:

$$
\widehat{\omega}:=\arg \max _{\omega} \mathcal{L}(\omega)
$$

\subsection{Computational implementation}

To estimate the parameters of the generalized linear model given by expression (9), we used the software $\mathrm{R}$. The "gnm" library includes the function gnm(). The iterative algorithm requires starting values for the parameters, which were obtained through the function nls() in the "nls" library. To estimate the corresponding model (12) for the second stage, we utilized the "tscount" library for discrete time dependent variables, in combination with the generalized linear models library "glm" .

To obtain confidence intervals for out-of-sample predictions, we approximated the quasi-Poisson likelihood with negative binomial distributions via a bootstrap of size 10,000, using the ciTools library in $\mathrm{R}$, as described by Haman (2017). The intervals are, thus, built as follows:

1. The model in equation (9) is fitted to obtain estimates $\widehat{\theta}$ and $\widehat{\operatorname{Cov}}(\widehat{\theta})$. The number of simulations is set at 10,000 .

2. Simulate 10,000 draws of the coefficients $\widehat{\theta}_{*} \sim N(\widehat{\theta}, \widehat{\operatorname{Cov}}(\widehat{\theta}))$.

3. Simulate $Y_{*} \mid \mathcal{F}_{t-1}$ from the response distribution using the following approximation:

$$
\text { Negative Binomial }\left(\widehat{\lambda_{t}}, \widehat{\lambda_{t}} /(\widehat{\phi}-1)\right) \text {. }
$$

4. Determine quantiles $\alpha / 2$ and $(1-\alpha / 2)$ of the simulated conditional responses. 
For the case where the out-of-sample predictions follow a Gaussian distribution, we approximated the confidence intervals via the Delta method. For the point estimate, we use a first-order Taylor expansion:

$$
\widehat{\lambda}_{t}=\lambda_{t}(\widehat{\theta}) \simeq \lambda_{t}\left(\theta_{0}\right)+\nabla \lambda_{t}\left(\theta_{0}\right)^{\prime}\left(\widehat{\theta}-\theta_{0}\right),
$$

where $\theta_{0}=\operatorname{plim} \widehat{\theta}$, and $\nabla \lambda_{t}\left(\theta_{0}\right)$ is the gradient of the new cases curve $\lambda_{t}$ evaluated at $\theta_{0}$. The variance, then, can be approximated with:

$$
\widehat{\operatorname{Var}}\left(\widehat{\lambda}_{t}\right) \simeq \nabla \lambda_{t}(\widehat{\theta})^{\prime} \operatorname{Cov}(\widehat{\theta}) \cdot \nabla \lambda_{t}(\widehat{\theta})
$$

Therefore, the resulting $(1-\alpha) \%$ confidence interval is given by:

$$
\left[\widehat{\lambda}_{t}-z_{1-\alpha / 2} \sqrt{\nabla \lambda_{t}(\widehat{\theta})^{\prime} \operatorname{Cov}(\widehat{\theta}) \cdot \nabla \lambda_{t}(\widehat{\theta})+\sigma^{2}}, \widehat{\lambda}_{t}+z_{1-\alpha / 2} \sqrt{\nabla \lambda_{t}(\widehat{\theta})^{\prime} \operatorname{Cov}(\widehat{\theta}) \cdot \nabla \lambda_{t}(\widehat{\theta})+\sigma^{2}}\right]
$$

\subsection{Forecast accuracy evaluation}

We are interested in comparing the forecast accuracy of these models. In order to choose models, it is common practice (see Gitto et al. (2021); Zreiq et al. (2020); Dal Molin Ribeiro et al. (2020) as examples) to identify a training sample, where the training data is used to estimate the parameters of a forecasting method, and a test sample, that is used to evaluate its accuracy. Two popularly used scale-dependent measures are the mean absolute error (MAE) and the root mean square error (RMSE), whereas the most common measure on percentage errors (thus, unit-free) is the mean absolute percentage error (MAPE):

$$
\begin{aligned}
\text { MAE } & =h^{-1} \sum_{j=1}^{h}\left|y_{t+j}-\widehat{y}_{t+j}\right| \\
\text { MAPE } & =h^{-1} \sum_{j=1}^{h}\left|y_{t+j}-\widehat{y}_{t+j}\right| / y_{t+j} \\
\text { RMSE } & =h^{-1} \sum_{j=1}^{h}\left(y_{t+j}-\widehat{y}_{t+j}\right)^{2}
\end{aligned}
$$

where $\widehat{y}_{t+j}$ denotes its $j^{t h}$ predictor based on information up to period $t$.

From these measures, we can choose the estimates that yield their lowest values in the out-of-sample forecasting window. To better validate the estimates and to evaluate the flexibility of the proposed models, we considered two different cut-off points for the training sample (April 15th 2021, and May 15th 2021), and three different corresponding forecasting horizons (3, 7 and 14 days ahead).

\section{Results and Discussion}

In our analyses, we first considered data from January 1st (the day after the announcement of the issue of the vacation permits, which itself marked the start of the second wave of the pandemic in Chile) until April 15th 2021, to make forecasts from April 16th onward. Subsequently, we repeated the same analyses, now using a sample including one extra month, until May 15th 2021, to make forecasts from May 16th onward. Our rationale was that, for each of the two cutoff points, given the nonlinear dynamics 
of the disease, the performance of each model would be different as well. As such, this would highlight the complementary aspect of each modeling approach.

The observed count of new daily cases is displayed in Figure 1, and the utilization of COVID-19 ICU beds is shown in Figure 2. The vertical lines in both cases denote the two cutoff points considered. Figure 1 shows that the number of new cases peaks slightly after the first cutoff point, stabilizing shortly after. On the other hand, we can observe in Figure 2 that the utilization of ICU beds (in spite of being a stock variable, not a flow variable) also can be modeled as a logistic growth curve, having reached a peak in late April, slowly decreasing after that date.

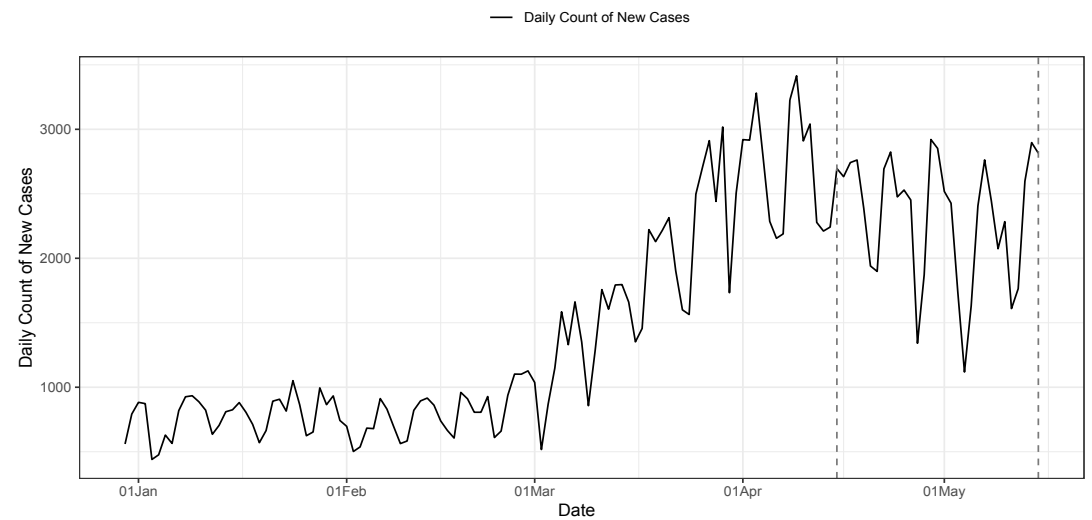

Figure 1: Daily count of new COVID-19 diagnosed cases in Santiago, Chile, between January 1st and May 15th 2021.

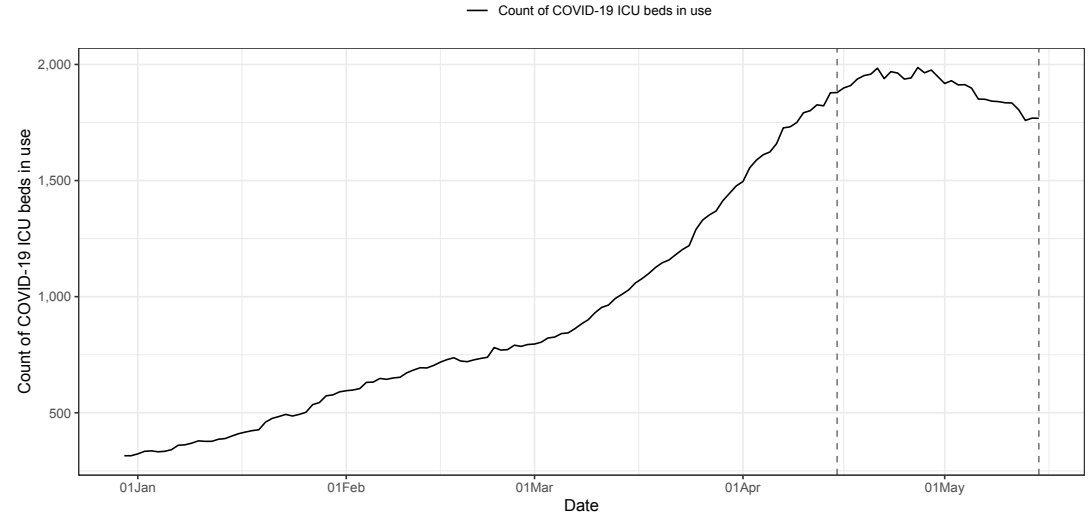

Figure 2: Daily utilization of ICU beds with COVID-19 patients in Santiago, Chile, from January 1st to May 15th 2021 
Table 1: First-stage estimated parameters' $p$-values, cutoff at 2021-04-15

\begin{tabular}{c|rr|rr|rr}
\hline \multirow{2}{*}{ Parameter } & \multicolumn{2}{|c|}{ Weibull } & \multicolumn{2}{c|}{ Logistic } & \multicolumn{2}{c}{ Harvey } \\
& Q-Poisson & Gaussian & Q-Poisson & Gaussian & Q-Poisson & Gaussian \\
\hline$\delta_{0}$ & $<0.0001$ & $<0.0001$ & $<0.0001$ & $<0.0001$ & 0.892 & 0.659 \\
$\vartheta_{1}$ & 0.9376 & 0.9930 & $<0.0001$ & $<0.0001$ & $<0.0001$ & $<0.0001$ \\
$\vartheta_{2}$ & $<0.0001$ & $<0.0001$ & $<0.0001$ & $<0.0001$ & $<0.0001$ & $<0.0001$ \\
$\vartheta_{3}$ & 0.7635 & 0.9760 & $<0.0001$ & $<0.0001$ & 0.0002 & 0.0091 \\
$\psi$ & 0.0002 & $<0.0001$ & 0.104 & 0.0051 & $<0.0001$ & $<0.0001$ \\
$\beta_{1}$ & 0.3351 & 0.2337 & 0.1715 & 0.0796 & 0.2497 & 0.1882 \\
$\beta_{2}$ & $<0.0001$ & $<0.0001$ & $<0.0001$ & $<0.0001$ & $<0.0001$ & $<0.0001$ \\
$\beta_{3}$ & $<0.0001$ & $<0.0001$ & $<0.0001$ & $<0.0001$ & $<0.0001$ & $<0.0001$ \\
$\beta_{4}$ & 0.0120 & 0.0076 & $<0.0001$ & $<0.0001$ & 0.1037 & 0.0551 \\
$\beta_{5}$ & $<0.0001$ & $<0.0001$ & $<0.0001$ & $<0.0001$ & 0.0036 & $<0.0001$ \\
$\beta_{6}$ & 0.0002 & $<0.0001$ & $<0.0001$ & $<0.0001$ & 0.0066 & 0.0003 \\
$\beta_{7}$ & 0.0364 & 0.0246 & $<0.0001$ & 0.0046 & 0.0066 & 0.0518 \\
\hline
\end{tabular}

Table 2: First-stage estimated parameters' $p$-values, cutoff at 2021-05-15

\begin{tabular}{c|rr|rr|rr}
\hline \multirow{2}{*}{ Parameter } & \multicolumn{2}{|c|}{ Weibull } & \multicolumn{2}{c|}{ Logistic } & \multicolumn{2}{c}{ Harvey } \\
& Q-Poisson & Gaussian & Q-Poisson & Gaussian & Q-Poisson & Gaussian \\
\hline$\delta_{0}$ & $<0.0001$ & $<0.0001$ & $<0.0001$ & $<0.0001$ & 0.0317 & 0.225 \\
$\vartheta_{1}$ & 0.925 & $<0.0001$ & $<0.0001$ & $<0.0001$ & $<0.0001$ & $<0.0001$ \\
$\vartheta_{2}$ & 0.825 & $<0.0001$ & $<0.0001$ & $<0.0001$ & 0.2269 & $<0.0001$ \\
$\vartheta_{3}$ & $<0.0001$ & $<0.0001$ & $<0.0001$ & $<0.0001$ & 0.8580 & $<0.0001$ \\
$\psi$ & $<0.0001$ & 0.1559 & - & - & $<0.0001$ & 0.625 \\
$\beta_{1}$ & 0.3049 & $<0.0001$ & 0.1910 & 0.1465 & 0.1457 & 0.1033 \\
$\beta_{2}$ & $<0.0001$ & $<0.0001$ & $<0.0001$ & $<0.0001$ & $<0.0001$ & $<0.0001$ \\
$\beta_{3}$ & $<0.0001$ & $<0.0001$ & $<0.0001$ & $<0.0001$ & $<0.0001$ & $<0.0001$ \\
$\beta_{4}$ & 0.0009 & $<0.0001$ & $<0.0001$ & $<0.0001$ & 0.0337 & 0.0171 \\
$\beta_{5}$ & $<0.0001$ & $<0.0001$ & $<0.0001$ & $<0.0001$ & 0.0050 & 0.0014 \\
$\beta_{6}$ & 0.0033 & $<0.0001$ & $<0.0001$ & $<0.0001$ & 0.0753 & 0.0314 \\
$\beta_{7}$ & 0.1214 & 0.0382 & 0.0621 & 0.0349 & 0.4362 & 0.5049 \\
\hline
\end{tabular}

\subsection{First stage results}

We fit the different curves we proposed above to predict the new cases of COVID-19 in Santiago, Chile. Tables 1 and 2 display the statistical significance of the estimated parameters for each of the parameters considering both cutoff points.

Looking at Table 1, under the April 15th cutoff scenario, it is plausible to observe that for a 5\% significance level, the lockdown intervention parameter, $\psi$, is not statistically significant for the QuasiPoisson logistic curve, whereas the offset parameter $\delta_{0}$ is statistically not different from zero in the Harvey curve cases. From Table 2, using the May 15th cutoff, it is possible to see that $\psi$ is statistically insignificant for the Gaussian Harvey and Weibull cases. In the case of the two Richards/logistic curves, the $\psi$ parameter was not properly estimated, because the effect of the entire term $\exp \left(\psi I_{t}\right)$ was estimated to be statistically zero for those curves (meaning that the corresponding estimated value of $\psi$ would be as close to $-\infty$ as possible).

We display our estimated epidemiological curves in Figures 3-4 (Quasi-Poisson and Gaussian, respectively, for the April 15th cutoff) and Figures 5-6 (Quasi-Poisson and Gaussian, respectively, for the May 15th cutoff). Additionally, Tables 3 and 4 contain our forecasting accuracy measures for each respective 
cutoff.

Analyzing our out-of-sample performance, for the April 15th cutoff, the model that best performs is the Gaussian Richards/logistic curve. This curve yields the lowest values of MAE, MAPE and RMSE considering forecasting horizons of 3, 7 and 14 days ahead. The results of the Quasi-Poisson Richards/logistic curve are not qualitatively dissimilar. The Harvey and the Weibull curves, instead, performed substantially worse, in that they overestimated the forecasts compared to the actual values.

For the May 15th cutoff, our results are in a way opposite to what we obtained for the first cutoff: the Richards/logistic curves continued estimating a downward trajectory, yielding systematic underestimations of the count of new cases for this new period. The Gaussian Harvey and Weibull curves also underestimated the new cases compared to the actual realizations for the forecasting horizon. As one can see in Figure 5, the Quasi-Poisson Weibull and Harvey curves yield reasonable estimates for the forecasting horizon. This is also supported by the performance measures: the best MAE/MAPE/RMSE was obtained with the Quasi-Poisson Weibull curve when considering a 3-to-7 day day horizon, whereas the best measures for the 14-day horizon were obtained with the Quasi-Poisson Harvey curve.

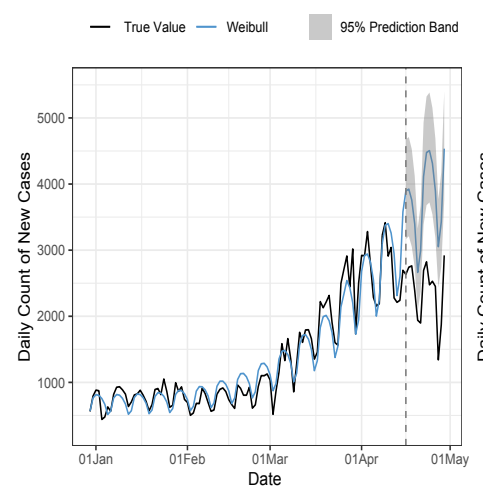

(a)

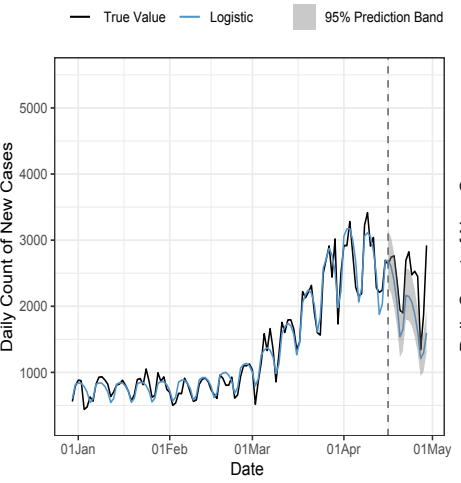

(b)

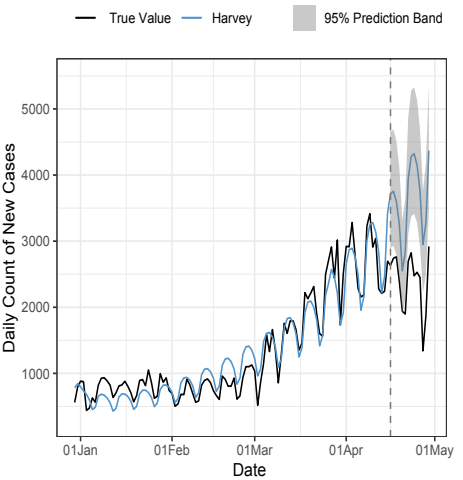

(c)

Figure 3: Fitted curves for daily count of new cases, Quasi-Poisson distribution, cutoff at 2021-04-15, and out-of-sample forecasts for 2021-04-16 to 2021-04-29

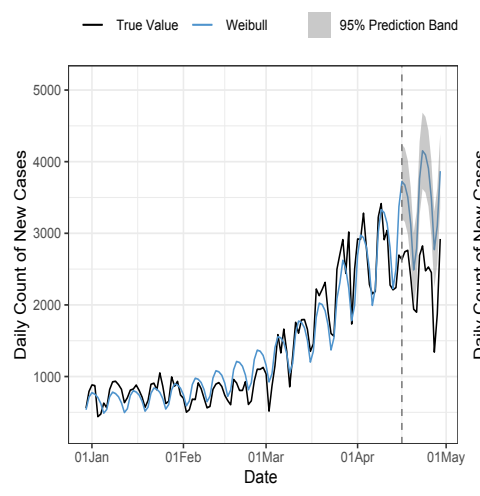

(a)

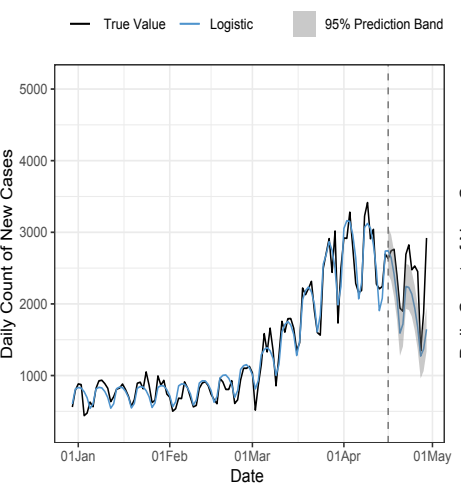

(b)

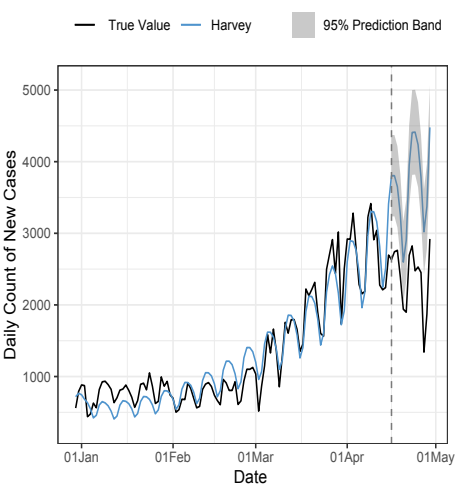

(c)

Figure 4: Fitted curves for daily count of new cases, Gaussian distribution, cutoff at 2021-04-15, and out-of-sample forecasts for 2021-04-16 to 2021-04-29 

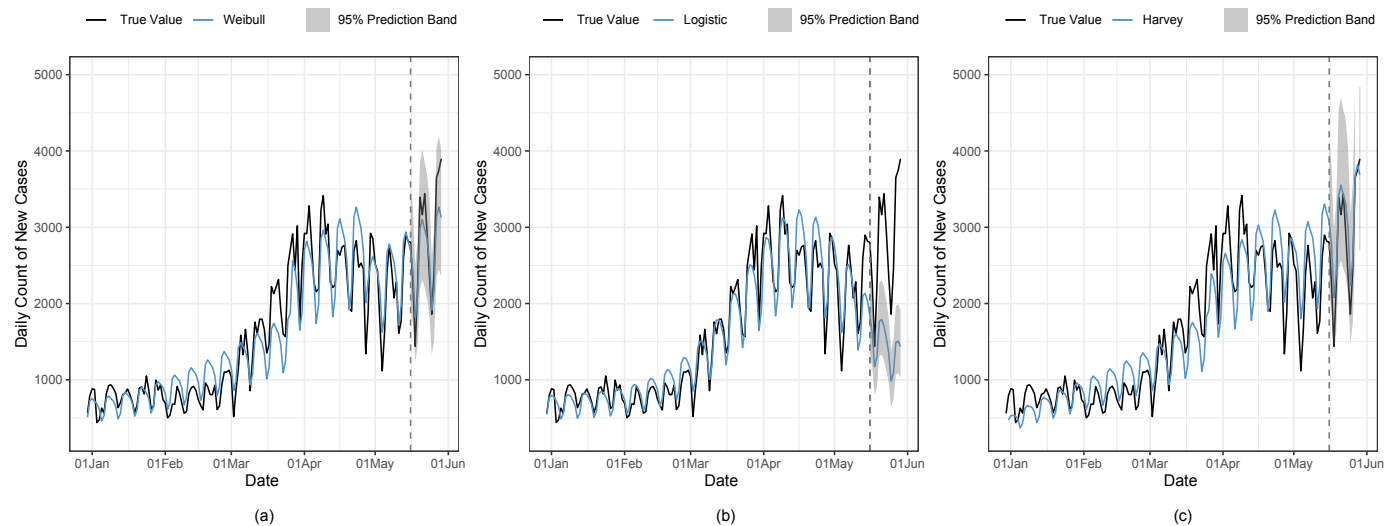

Figure 5: Fitted curves for daily count of new cases, Quasi-Poisson distribution, cutoff at 2021-05-15, and out-of-sample forecasts for 2021-05-16 to 2021-05-29
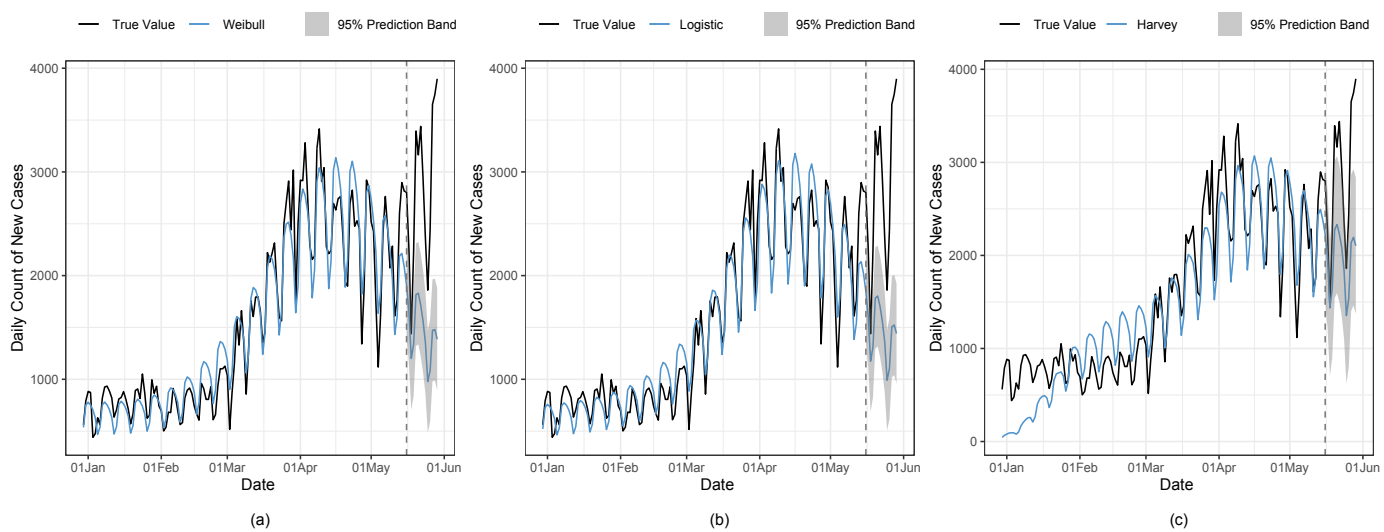

Figure 6: Fitted curves for daily count of new cases, Gaussian distribution, cutoff at 2021-05-15, and out-of-sample forecasts for 2021-05-16 to 2021-05-29

Table 3: Performance measures of the forecasts cutoff at 2021-04-15

\begin{tabular}{c|c|ccc|ccc|ccc}
\hline \multirow{2}{*}{ Data } & \multirow{2}{*}{ Measure } & \multicolumn{3}{|c|}{ Three-day forecast } & \multicolumn{3}{c|}{ Seven-day forecast } & \multicolumn{3}{c}{ Fourteen-day forecast } \\
\cline { 3 - 12 } & & Weibull & Logistic & Harvey & Weibull & Logistic & Harvey & Weibull & Logistic & Harvey \\
\hline \multirow{3}{*}{ Q-Poisson } & MAE & 1146 & 194 & 978 & 1099 & 299 & 941 & 1392 & 478 & 1234 \\
& MAPE & 0.424 & 0.071 & 0.361 & 0.453 & 0.126 & 0.388 & 0.609 & 0.196 & 0.542 \\
& RMSE & 1152 & 238 & 983 & 1118 & 334 & 960 & 1435 & 575 & 1280 \\
\hline \multirow{3}{*}{ Gaussian } & MAE & 922 & 184 & 1040 & 859 & 261 & 987 & 1070 & 426 & 1301 \\
& MAPE & 0.341 & 0.067 & 0.385 & 0.354 & 0.110 & 0.407 & 0.473 & 0.173 & 0.572 \\
& RMSE & 932 & 217 & 1047 & 878 & 289 & 1005 & 1109 & 527 & 1350 \\
\hline
\end{tabular}


Table 4: Performance measures of the forecasts cutoff at 2021-05-15

\begin{tabular}{c|c|ccc|ccc|ccc}
\hline \multirow{3}{*}{ Data } & \multirow{2}{*}{ Measure } & \multicolumn{3}{|c|}{ Three-day forecast } & \multicolumn{3}{c|}{ Seven-day forecast } & \multicolumn{3}{c}{ Fourteen-day forecast } \\
\cline { 3 - 12 } & & Weibull & Logistic & Harvey & Weibull & Logistic & Harvey & Weibull & Logistic & Harvey \\
\hline \multirow{3}{*}{ Q-Poisson } & MAE & 219 & 627 & 477 & 252 & 1079 & 288 & 296 & 1360 & 274 \\
& MAPE & 0.126 & 0.272 & 0.257 & 0.104 & 0.379 & 0.139 & 0.103 & 0.457 & 0.122 \\
& RMSE & 248 & 688 & 500 & 294 & 1186 & 364 & 360 & 1492 & 346 \\
\hline \multirow{3}{*}{ Gaussian } & MAE & 580 & 624 & 292 & 1039 & 1072 & 661 & 1346 & 1351 & 876 \\
& MAPE & 0.251 & 0.271 & 0.113 & 0.363 & 0.377 & 0.221 & 0.451 & 0.454 & 0.285 \\
& RMSE & 640 & 686 & 375 & 1151 & 1178 & 772 & 1488 & 1482 & 1006 \\
\hline
\end{tabular}

Tables 5 and 6 show our forecasts for some specific dates, between April 16th and 29th for the first cutoff, and between May 16th and 29th for the second, including 95\% confidence forecasting intervals for each curve. From Table 5, with the April 15th cutoff, we can see that the short-term forecast intervals using the Quasi-Poisson (up to 3 days ahead) and Gaussian (up to 2 days ahead) logistic curves contain the true values, underestimating the counts in the longer run, whereas the Harvey and Weibull curves always overestimate the counts. For the May 15th cutoff, we can see from Table 6 that the Quasi-Poisson forecasts using the Harvey and Weibull cases perform well, containing the true values in the 7- and 14days-ahead forecast intervals, while the logistic curves underestimate in about $60 \%$ of the true values in the 14-day forecast horizon.

Table 5: Forecasts for new cases with 95\% confidence forecasting intervals. 2021-04-15 cutoff.

\begin{tabular}{|c|c|c|c|c|c|c|c|}
\hline \multirow[b]{2}{*}{ Date } & \multirow[b]{2}{*}{ Actual } & \multicolumn{2}{|c|}{ Weibull } & \multicolumn{2}{|c|}{ Logistic } & \multicolumn{2}{|c|}{ Harvey } \\
\hline & & Q-Poisson & Gaussian & Q-Poisson & Gaussian & Q-Poisson & Gaussian \\
\hline 2021-04-16 & 2633 & $\begin{array}{c}3895.7 \\
(3186,4686)\end{array}$ & $\begin{array}{c}3722.5 \\
(2453,5246)\end{array}$ & $\begin{array}{c}2689.2 \\
(2265,3144)\end{array}$ & $\begin{array}{c}2742.1 \\
(2418,3065)\end{array}$ & $\begin{array}{c}3718.2 \\
(2876,4662)\end{array}$ & $\begin{array}{c}3804.3 \\
(3236,4373)\end{array}$ \\
\hline 2021-04-17 & 2742 & $\begin{array}{c}3922.6 \\
(3211,4711)\end{array}$ & $\begin{array}{c}3674.3 \\
(3159,4190)\end{array}$ & $\begin{array}{c}2598.8 \\
(2184,3058)\end{array}$ & $\begin{array}{c}2645.1 \\
(2324,2966)\end{array}$ & $\begin{array}{c}3753.3 \\
(2903,4686)\end{array}$ & $\begin{array}{c}3804.2 \\
(3236,4372)\end{array}$ \\
\hline 2021-04-18 & 2762 & $\begin{array}{c}3757.7 \\
(3059,4541)\end{array}$ & $\begin{array}{c}3505.1 \\
(2990,4021\end{array}$ & $\begin{array}{c}2379.6 \\
(1991,2808)\end{array}$ & $\begin{array}{c}2414.9 \\
(2095,2734)\end{array}$ & $\begin{array}{c}3598.1 \\
(2787,4528)\end{array}$ & $\begin{array}{c}3649.6 \\
(3081,4218)\end{array}$ \\
\hline 2021-04-22 & 2693 & $\begin{array}{c}4114.8 \\
(3380,4936)\end{array}$ & $\begin{array}{c}3762.1 \\
(3248,4277)\end{array}$ & $\begin{array}{c}2165.3 \\
(1801,2571)\end{array}$ & $\begin{array}{c}2243.2 \\
(1929,2557)\end{array}$ & $\begin{array}{c}3936.9 \\
(3082,4906)\end{array}$ & $\begin{array}{c}3950.5 \\
(3382,4519)\end{array}$ \\
\hline 2021-04-29 & 2921 & $\begin{array}{c}4534.8 \\
(3786,5380)\end{array}$ & $\begin{array}{c}3867.7 \\
(3350,4386)\end{array}$ & $\begin{array}{c}1596.9 \\
(1285,1940)\end{array}$ & $\begin{array}{c}1645.7 \\
(1339,1953)\end{array}$ & $\begin{array}{c}4369.4 \\
(3478,5403)\end{array}$ & $\begin{array}{c}4478.5 \\
(3890,5067)\end{array}$ \\
\hline
\end{tabular}

\subsection{Second stage results}

Now, we move onto our main variable of interest: the daily utilization of ICU beds with COVID-19 patients. We forecast this variable estimating a mean demand model specified through equation (12).

In choosing of our best forecasting model, we face a trade-off between improving the predictive ability of the model and minimizing its residual correlation. We analyzed several models, considering different choices of the corresponding lag lengths $p$ and $q$, and we obtained the best results by parameterizing equation (12) with the specification presented as equation (14):

$$
\mu_{t}=\alpha_{0}+\alpha_{3} Y_{t-3}+\eta_{1} \ln \left(X_{1, t}\right)+\eta_{2} X_{2, t}
$$


Table 6: Forecasts for new cases with 95\% confidence forecasting intervals. 2021-05-15 cutoff.

\begin{tabular}{|c|c|c|c|c|c|c|c|}
\hline \multirow[b]{2}{*}{ Date } & \multirow[b]{2}{*}{ Actual } & \multicolumn{2}{|c|}{ Weibull } & \multicolumn{2}{|c|}{ Logistic } & \multicolumn{2}{|c|}{ Harvey } \\
\hline & & Q-Poisson & Gaussian & Q-Poisson & Gaussian & Q-Poisson & Gaussian \\
\hline $2021-05-16$ & 2804 & $\begin{array}{c}2693 \\
(1961,3547)\end{array}$ & $\begin{array}{c}1903.9 \\
(1406,2402)\end{array}$ & $\begin{array}{c}1840.5 \\
(1366,2384)\end{array}$ & $\begin{array}{c}1846.1 \\
(1356,2336)\end{array}$ & $\begin{array}{c}3078.2 \\
(2175,4162)\end{array}$ & $\begin{array}{c}2225.9 \\
(1487,2965)\end{array}$ \\
\hline 2021-05-17 & 2260 & $\begin{array}{c}2428.2 \\
(1744,3212)\end{array}$ & $\begin{array}{c}1656.8 \\
(1159,2154)\end{array}$ & $\begin{array}{c}1611.1 \\
(1174,2115)\end{array}$ & $\begin{array}{c}1609.8 \\
(1120,2099)\end{array}$ & $\begin{array}{c}2775.9 \\
(1920,3768)\end{array}$ & $\begin{array}{c}1963.6 \\
(1225,2702)\end{array}$ \\
\hline 2021-05-18 & 1439 & $\begin{array}{c}1817.5 \\
(1244,2533)\end{array}$ & $\begin{array}{c}1201.3 \\
(705,1698)\end{array}$ & $\begin{array}{c}1170.9 \\
(797,1604)\end{array}$ & $\begin{array}{c}1174.2 \\
(685,1663)\end{array}$ & $\begin{array}{c}2078.5 \\
(1338,2949\end{array}$ & $\begin{array}{c}1441.6 \\
(704,2179)\end{array}$ \\
\hline $2021-05-22$ & 3439 & $\begin{array}{c}2971.4 \\
(2193,3865)\end{array}$ & $\begin{array}{c}1716.5 \\
(1223,2211)\end{array}$ & $\begin{array}{c}1703.5 \\
(1243,2231)\end{array}$ & $\begin{array}{c}1704.8 \\
(1218,2192)\end{array}$ & $\begin{array}{c}3424.2 \\
(2466,4526)\end{array}$ & $\begin{array}{c}2232.3 \\
(1497,2967)\end{array}$ \\
\hline $2021-05-29$ & 3898 & $\begin{array}{c}3127.5 \\
(2339,4018)\end{array}$ & $\begin{array}{c}1386.1 \\
(895,1877)\end{array}$ & $\begin{array}{c}1437.5 \\
(1019,1907)\end{array}$ & $\begin{array}{c}1442.1 \\
(958,1926)\end{array}$ & $\begin{array}{c}3683.7 \\
(2685,4860)\end{array}$ & $\begin{array}{c}2103.3 \\
(1371,2836)\end{array}$ \\
\hline
\end{tabular}

where, as before, $\mu_{t}$ is the mean demand rate of the process on day $t ; Y_{t}$ is the utilization of COVID-19 ICU beds on day $t$; covariate $X_{1, t}=\sum_{i=1}^{t-1} Y_{i}$ is a Harvey-like component measuring an accumulation of past demands; and covariate $X_{2, t}=\frac{1}{7} \sum_{i=t-8}^{t-1} X_{i}$ is the average of the daily count of new cases over the past 7 days.

Figure 7 contains the fitted curve overlaid on the actual data. Panel (a) shows the fit of the model for the April 15th cutoff, and (b) does the same for the May 15th cutoff. In both cases, the model captures remarkably well the empirical dynamics of the utilization of ICU beds. Tables 7 and 8 display the estimated parameters of the model for each respective cutoff point, along with standard deviations and $p$-values. All estimated parameters are statistically different from zero at the 5\% significance level.

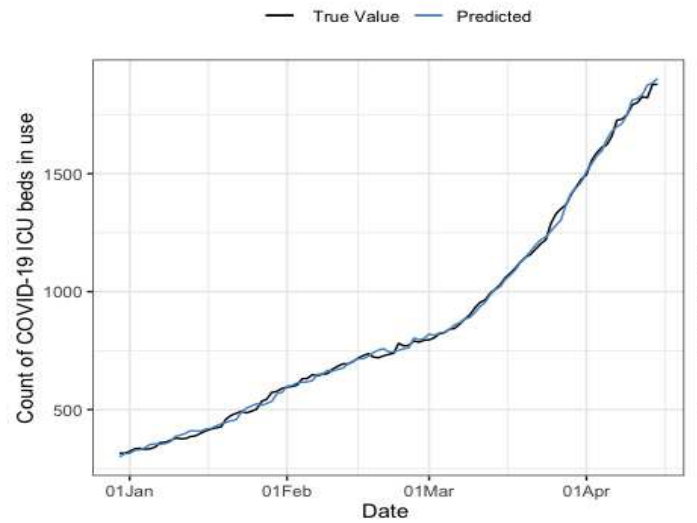

(a)

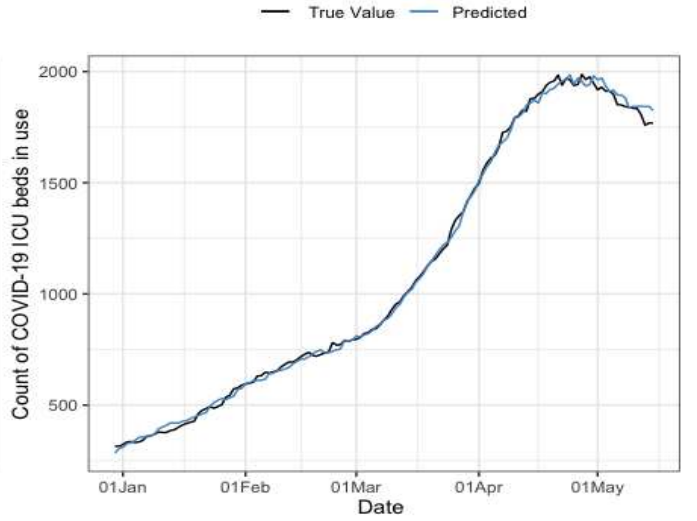

(b)

Figure 7: Utilization of COVID-19 ICU beds. Model fitted using cutoff dates of 2021-04-15 (panel (a)) and 2021-05-15 (panel (b)).

Figures 8 and 9 display the model forecasts two weeks after the April 15th cutoff date, totaling six estimated curves from the first stage, working as inputs for the second stage. Figures 10 and 11 show the 
Table 7: Estimated parameters, COVID-19 ICU beds utilization model, 2021-04-15 cutoff

\begin{tabular}{ccccc}
\hline Parameter & Estimate & Std. Error & z-value & $p$-value \\
\hline$\alpha_{0}$ & -69.76440 & 28.93183 & -2.411 & 0.0159 \\
$\alpha_{3}$ & 0.93570 & 0.03005 & 31.136 & $<0.0001$ \\
$\eta_{1}$ & 8.87023 & 3.84742 & 2.306 & 0.0211 \\
$\eta_{2}$ & 0.06096 & 0.01408 & 4.329 & $<0.0001$ \\
\hline
\end{tabular}

Table 8: Estimated parameters, COVID-19 ICU beds utilization model, 2021-05-15 cutoff

\begin{tabular}{ccccc}
\hline Parameter & Estimate & Std. Error & z-value & $p$-value \\
\hline$\alpha_{0}$ & -117.18608 & 24.49111 & -4.785 & $<0.0001$ \\
$\alpha_{1}$ & 16.09359 & 3.07295 & 5.237 & $<0.0001$ \\
$\alpha_{2}$ & 0.84742 & .01695 & 50.008 & $<0.0001$ \\
$\alpha_{3}$ & 0.09619 & 0.01051 & 9.149 & $<0.0001$ \\
\hline
\end{tabular}

analogous results for the May 15th cutoff date. Tables 9 and 10 show the performance measures (MAE, MAPE, RMSE) for each estimated model, considering each cutoff point.

We can see that, as expected, the performance of each model in this second stage is directly affected by the performance of the first stage input models. From figures 8 and 9 from the April cutoff point, we can observe that when the model for new cases only slightly overestimates the count compared to the actual results (as it is the case of the Logistic curve), the ICU beds demand model provides an appropriate fit even for the 14-days-ahead forecast horizon. On the other hand, the corresponding forecasts using Weibull's or Harvey's curves, where the count of new cases is substantially overestimated, result in an also excessively high forecast of ICU beds demand. Table 9 also supports the aforementioned graphical analysis, with better goodness-of-fit indicators for the Quasi-Poisson Logistic curves, followed by the Gaussian Logistic case.

Using now the May 15th cutoff point, figures 10 and 11 display that the best out-of-sample predictions for new cases are obtained using a Gaussian Harvey and a Quasi-Poisson Weibull curve, with the Gaussian Harvey curve for new cases generating 95\% forecasting intervals for the demand of ICU beds that ex-post contain the true values. On the other hand, the Gaussian Weibull and both Logistic curves yielded excessively low forecasts of the new cases count compared to the true values, which in turn systematically underestimated the demand of ICU beds. This underestimation result, from a policy standpoint, is much more problematic when compared to overestimation: when one overestimates demand, the system builds capacity unnecessarily, whereas, when demand is underestimated, we risk facing the inability to save lives. Please note that the goodness-of-fit measures collected in table 10 do not take that differential cost into consideration, demonstrating how using goodness-of-fit measures by themselves, without taking into account the graphical representation and the differential cost effect of erring on either side of the forecast, is insufficient for this type of policy analyses.

Tables 11 and 12 present some forecast values ahead for each cutoff point under consideration, providing support to our results as discussed above. 


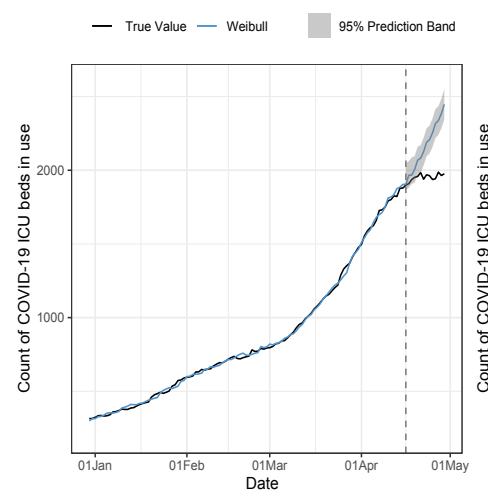

(a)
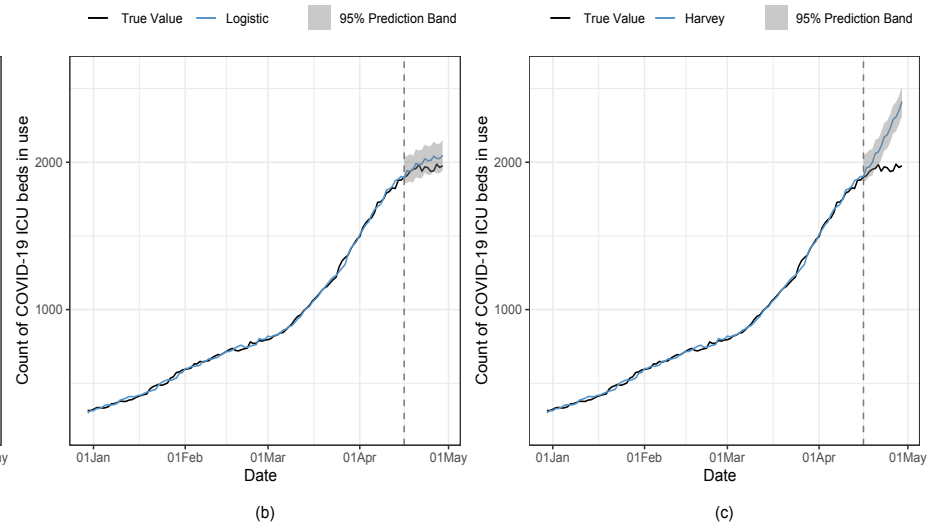

Figure 8: Forecast Performance for ICU beds models, Quasi-Poisson distribution, April 15th cutoff
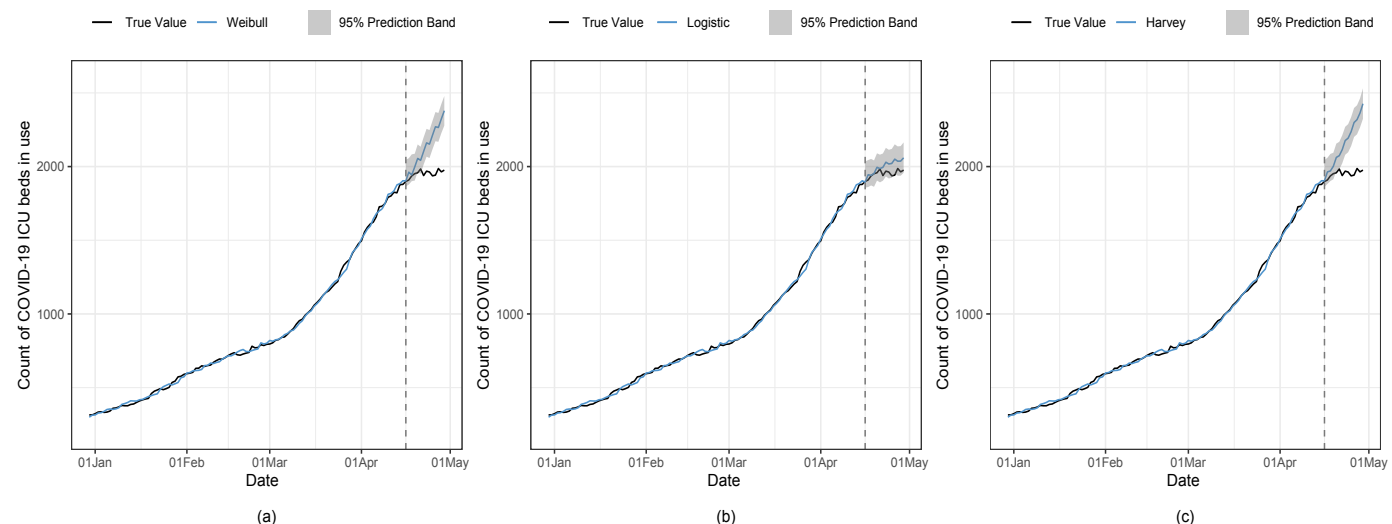

Figure 9: Forecast Performance for ICU beds models, Gaussian distribution, April 15th cutoff
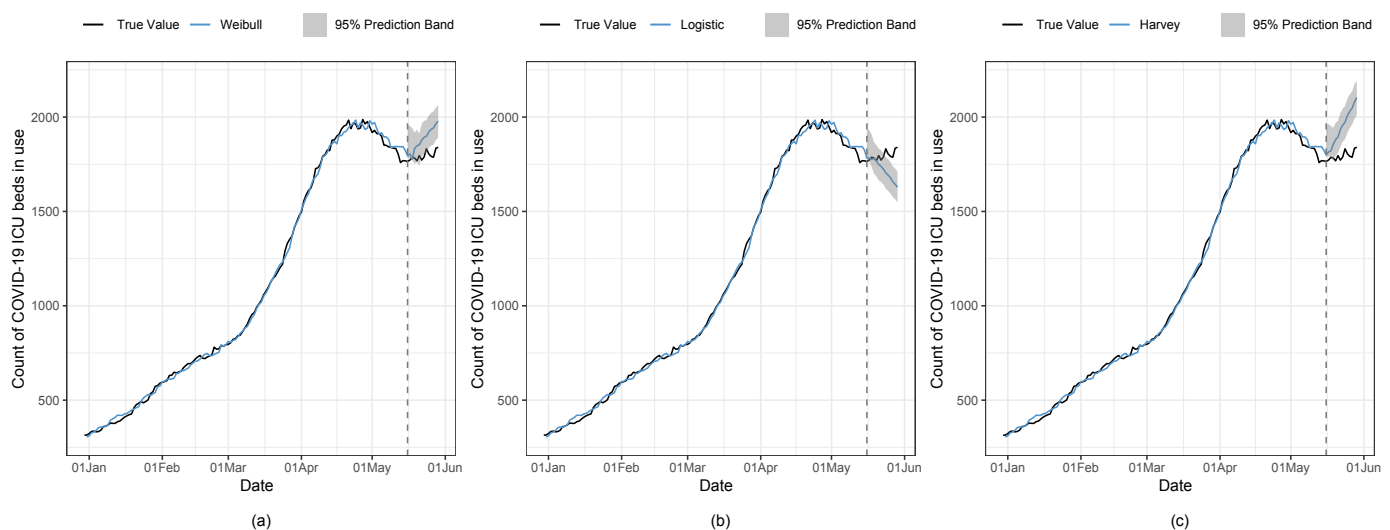

Figure 10: Forecast Performance for ICU beds models, Quasi-Poisson distribution, May 15th cutoff 


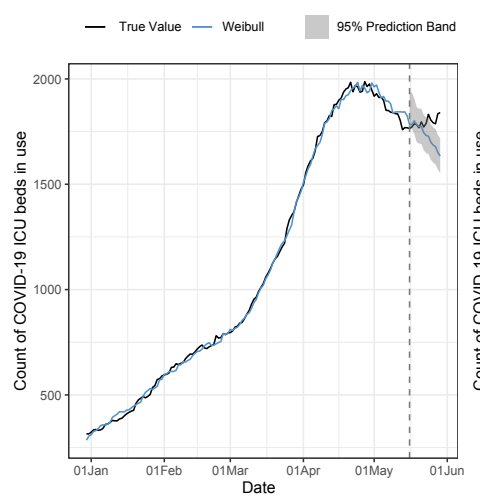

(a)

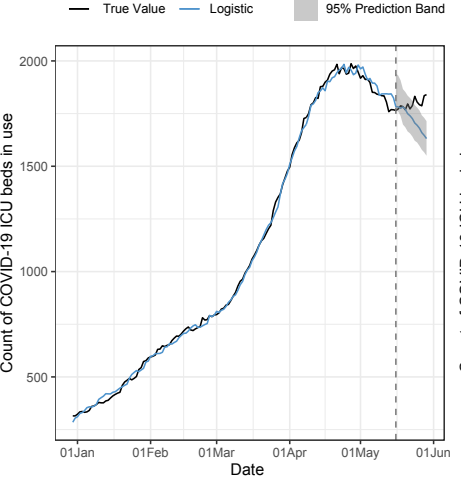

(b)

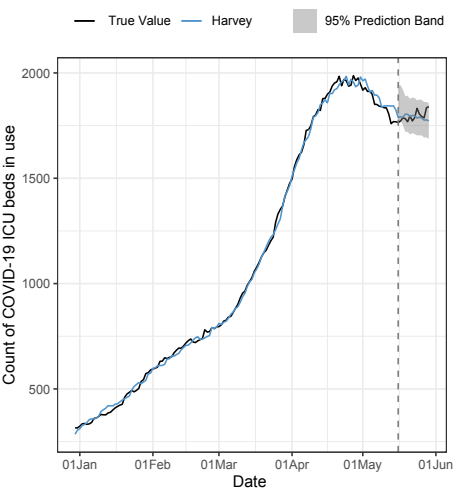

(c)

Figure 11: Forecast Performance for ICU beds models, Gaussian distribution, May 15th cutoff

Table 9: Performance measures for ICU beds utilization models, April 15th cutoff

\begin{tabular}{c|c|ccc|ccc|cccc}
\hline \multirow{3}{*}{ Data } & \multirow{2}{*}{ Measure } & \multicolumn{3}{c|}{ Three-day forecast } & \multicolumn{3}{c|}{ Seven-day forecast } & \multicolumn{3}{c}{ Fourteen-day forecast } \\
\cline { 3 - 12 } & & Weibull & Logistic & Harvey & Weibull & Logistic & Harvey & Weibull & Logistic & Harvey \\
\hline \multirow{3}{*}{ Q-Poisson } & MAE & 30 & 14 & 30 & 77 & 18 & 71 & 210 & 41 & 193 \\
& MAPE & 0.016 & 0.007 & 0.016 & 0.040 & 0.010 & 0.036 & 0.107 & 0.021 & 0.098 \\
& RMS & 37 & 20 & 36 & 95 & 27 & 87 & 258 & 51 & 238 \\
\hline \multirow{3}{*}{ Gaussian } & MAE & 21 & 15 & 31 & 61 & 20 & 73 & 175 & 47 & 199 \\
& MAPE & 0.011 & 0.008 & 0.016 & 0.031 & 0.010 & 0.03 & 0.089 & 0.024 & 0.102 \\
& RMS & 31 & 21 & 37 & 80 & 29 & 90 & 219 & 57 & 245 \\
\hline
\end{tabular}

Table 10: Performance measures for ICU beds utilization models, May 15th cutoff

\begin{tabular}{c|c|ccc|ccc|cccc}
\hline \multirow{3}{*}{ Data } & \multirow{2}{*}{ Measure } & \multicolumn{3}{c}{ Three-day forecast } & \multicolumn{3}{c|}{ Seven-day forecast } & \multicolumn{3}{c}{ Fourteen-day forecast } \\
\cline { 3 - 13 } & & Weibull & Logistic & Harvey & Weibull & Logistic & Harvey & Weibull & Logistic & Harvey \\
\hline \multirow{3}{*}{ Q-Poisson } & MAE & 24 & 13 & 37 & 52 & 18 & 80 & 87 & 77 & 148 \\
& MAPE & 0.014 & 0.007 & 0.021 & 0.029 & 0.010 & 0.045 & 0.048 & 0.042 & 0.082 \\
& RMSE & 26 & 13 & 37 & 60 & 24 & 91 & 98 & 103 & 170 \\
\hline \multirow{3}{*}{ Gaussian } & MAE & 15 & 13 & 15 & 14 & 17 & 19 & 69 & 75 & 24 \\
& MAPE & 0.008 & 0.007 & 0.008 & 0.008 & 0.010 & 0.011 & 0.038 & 0.042 & 0.014 \\
& RMSE & 15 & 13 & 18 & 16 & 23 & 22 & 95 & 101 & 31 \\
\hline
\end{tabular}


Table 11: COVID-19 ICU beds utilization fitted model with 95\% confidence forecast intervals, April 15 th cutoff

\begin{tabular}{|c|c|c|c|c|c|c|c|}
\hline \multirow[b]{2}{*}{ Date } & \multirow[b]{2}{*}{ Actual } & \multicolumn{2}{|c|}{ Weibull } & \multicolumn{2}{|c|}{ Logistic } & \multicolumn{2}{|c|}{ Harvey } \\
\hline & & Q-Poisson & Gaussian & Q-Poisson & Gaussian & Q-Poisson & Gaussian \\
\hline 2021-04-16 & 1899 & $\begin{array}{c}1904.3 \\
(1866,2045)\end{array}$ & $\begin{array}{c}1902.8 \\
(1865,2046)\end{array}$ & $\begin{array}{c}1893.8 \\
(1855,2035)\end{array}$ & $\begin{array}{c}1894.3 \\
(1857,2035)\end{array}$ & $\begin{array}{c}1902.8 \\
(1863,2043)\end{array}$ & $\begin{array}{c}1903.5 \\
(1862,2044)\end{array}$ \\
\hline 2021-04-17 & 1909 & $\begin{array}{c}1965.7 \\
(1882,2062)\end{array}$ & $\begin{array}{c}1962.1 \\
(1878,2057)\end{array}$ & $\begin{array}{c}1943.7 \\
(1860,2044)\end{array}$ & $\begin{array}{c}1944.6 \\
(1861,2044)\end{array}$ & $\begin{array}{c}1962.7 \\
(1879,2061)\end{array}$ & $\begin{array}{c}1963.9 \\
(1882,2060)\end{array}$ \\
\hline 2021-04-18 & 1937 & $\begin{array}{c}1964.7 \\
(1906,2089)\end{array}$ & $\begin{array}{c}1943.2 \\
(1901,2083)\end{array}$ & $\begin{array}{c}1939.1 \\
(1872,2055)\end{array}$ & $\begin{array}{c}1940.2 \\
(1872,2052)\end{array}$ & $\begin{array}{c}1968.7 \\
(1902,2083)\end{array}$ & $\begin{array}{c}1970.3 \\
(1902,2086)\end{array}$ \\
\hline 2021-04-22 & 1939 & $\begin{array}{c}2126.1 \\
(2032,2219)\end{array}$ & $\begin{array}{c}2102.4 \\
(2013,2198)\end{array}$ & $\begin{array}{c}1989.8 \\
(1895,2085)\end{array}$ & $\begin{array}{c}1994.8 \\
(1904,2089)\end{array}$ & $\begin{array}{c}2109.8 \\
(2015,2203)\end{array}$ & $\begin{array}{c}2114.8 \\
(2022,2212)\end{array}$ \\
\hline 2021-04-29 & 1976 & $\begin{array}{c}2447.2 \\
(2347,2550)\end{array}$ & $\begin{array}{c}2379.1 \\
(2278,2479)\end{array}$ & $\begin{array}{c}2046.2 \\
(1945,2147)\end{array}$ & $\begin{array}{c}2059.4 \\
(1958,2164)\end{array}$ & $\begin{array}{c}2412.0 \\
(2313,2513)\end{array}$ & $\begin{array}{c}2426.4 \\
(2324,2531)\end{array}$ \\
\hline
\end{tabular}

Table 12: COVID-19 ICU beds utilization fitted model with 95\% confidence forecast intervals, May 15th cutoff

\begin{tabular}{|c|c|c|c|c|c|c|c|}
\hline \multirow[b]{2}{*}{ Date } & \multirow[b]{2}{*}{ Actual } & \multicolumn{2}{|c|}{ Weibull } & \multicolumn{2}{|c|}{ Logistic } & \multicolumn{2}{|c|}{ Harvey } \\
\hline & & Q-Poisson & Gaussian & Q-Poisson & Gaussian & Q-Poisson & Gaussian \\
\hline 2021-05-16 & 1765 & $\begin{array}{c}1794.1 \\
(1781,1954)\end{array}$ & $\begin{array}{c}1783.2 \\
(1771,1941)\end{array}$ & $\begin{array}{c}1782.4 \\
(1767,1939)\end{array}$ & $\begin{array}{c}1782.5 \\
(1770,1940)\end{array}$ & $\begin{array}{c}1799.4 \\
(1787,1955)\end{array}$ & $\begin{array}{c}1787.7 \\
(1772,1942)\end{array}$ \\
\hline 2021-05-17 & 1772 & $\begin{array}{c}1804.7 \\
(1783,1953)\end{array}$ & $\begin{array}{c}1783.3 \\
(1762,1934\end{array}$ & $\begin{array}{c}1781.8 \\
(1760,1932)\end{array}$ & $\begin{array}{c}1781.9 \\
(1759,1931)\end{array}$ & $\begin{array}{c}1814.8 \\
(1794,1967)\end{array}$ & $\begin{array}{c}1791.9 \\
(1771,1942)\end{array}$ \\
\hline 2021-05-18 & 1787 & $\begin{array}{c}1776.5 \\
(1771,1941)\end{array}$ & $\begin{array}{c}1801.0 \\
(1740,1910)\end{array}$ & $\begin{array}{c}1775.1 \\
(1739,1908)\end{array}$ & $\begin{array}{c}1775.2 \\
(1739,1908)\end{array}$ & $\begin{array}{c}1820.6 \\
(1784,1956)\end{array}$ & $\begin{array}{c}1789.0 \\
(1753,1921)\end{array}$ \\
\hline $2021-05-22$ & 1772 & $\begin{array}{c}1877.7 \\
(1793,1965)\end{array}$ & $\begin{array}{c}1745.2 \\
(1662,1829)\end{array}$ & $\begin{array}{c}1738.6 \\
(1655,1823)\end{array}$ & $\begin{array}{c}1739.6 \\
(1656,1824\end{array}$ & $\begin{array}{c}1933.3 \\
(1847,2019)\end{array}$ & $\begin{array}{c}1798.5 \\
(1714,1883)\end{array}$ \\
\hline 2021-05-29 & 1840 & $\begin{array}{c}1978.1 \\
(1890,2068)\end{array}$ & $\begin{array}{c}1633.6 \\
(1552,1718)\end{array}$ & $\begin{array}{c}1628.4 \\
(1548,1710)\end{array}$ & $\begin{array}{c}1631.3 \\
(1550,1714)\end{array}$ & $\begin{array}{c}2104.1 \\
(2014,2196)\end{array}$ & $\begin{array}{c}1772.7 \\
(1687,1856)\end{array}$ \\
\hline
\end{tabular}

\section{Conclusion}

Our ability to understand the COVID-19 epidemic is crucial in order to curb its spread. The main contribution of our study lays in the provision of an important and simple framework to inform public health decision-making designed to build ICU capacity in a timely manner, and in the meantime, also illustrating the usefulness of nonlinear modeling approaches to forecast the evolution of the disease when availability of data is limited.

Limitations of the study include the fact that our forecasts are based on three growth models with two different distributional assumptions (six estimated growth curves used as inputs for the ICU beds model), as it would be interesting to compare forecasts from several different alternative methods.

Additionally, it is important to remember that the forecasting methods here presented for both the demand for ICU beds and the number of new daily cases are devised for the short-term. In the longer run (i.e., beyond two weeks ahead), the forecasts obtained from these models could differ in a substantial way from the true observed data. However, as a decision support strategy, we have provided a useful, easy to understand decision support tool, that doesn't require extremely detailed data to provide reasonable short-term forecasts, enabling authorities and decision makers to make informed decisions. 


\section{Data accessibility}

Data and code used in this research are available from the corresponding author upon request. Raw data are available from the official website of the Chilean Ministry of Science (https://www.minciencia.gob.cl/covid19).

\section{CRediT author statement}

Authors' names listed alphabetically. BFQ, CV and MIV defined the research problem, designed the study, conducted the data analyses and wrote the text of the manuscript. All authors read and approved the final manuscript.

\section{Declaration of competing interests}

The authors declare that this research was conducted in the absence of any commercial or financial relationships that could be construed as a potential conflict of interest.

\section{Acknowledgments}

Bernardo F. Quiroga gratefully acknowledges funding from FONDECYT Initiation Grant \#11191014.

\section{References}

Agosto, A., Giudici, P., 2020. A Poisson Autoregressive Model to Understand COVID-19 Contagion Dynamics. Risks 8, 3.

Andersen, K.G., Rambaut, A., Lipkin, W.I., Holmes, E.C., Garry, R.F., 2020. The proximal origin of SARS-CoV-2. Nature Medicine 26, 4, 450-452.

Aviv-Sharon, E., Aharoni, A., 2020. Generalized logistic growth modeling of the COVID-19 pandemic in Asia. Infectious Disease Modelling 5, 502 - 509.

Barroilhet, A., Ortiz, R., Quiroga, B.F., Silva, M., 2021. Exploring Conflict of Interest in University Accreditation in Chile. Higher Education Policy 34, 1-19.

Biswas, B., Sengupta, P., Chatterjee, D., 2020. Examining the determinants of the count of customer reviews in peer-to-peer home-sharing platforms using clustering and count regression techniques. Decision Support Systems 135, 113324.

Bloom, J.D., Chan, Y.A., Baric, R.S., Bjorkman, P.J., Cobey, S., Deverman, B.E., Fisman, D.N., Gupta, R., Iwasaki, A., Lipsitch, M., Medzhitov, R., Neher, R.A., et al., 2021. Investigate the origins of COVID-19. Science 372, 6543, 694-694.

Canals, M., Cuadrado, C., Canals, A., Yohannessen, K., Lefio, L.A., Bertoglia, M.P., Eguiguren, P., Siches, I., Iglesias, V., Arteaga, O., 2020. Epidemic trends, public health response and health system capacity: the Chilean experience in four months of the COVID-19 pandemic. Pan American Journal of Public Health 44, e99, 1-8.

Chen, D.G., Chen, X., Chen, J., 2020. Reconstructing and Forecasting the COVID-19 Epidemic in the US Using a 5-Parameter Logistic Growth Model. Global Health Research and Policy 5, 25, 1-7.

Chilean Ministry of Health, 2020. COVID-19: Gobierno anuncia permiso de vacaciones para comunas en Transición [in Spanish]. URL: https://www.minsal.cl/covid-19-gobierno-anuncia-permiso-de-vacaciones-para-comunas-en-transicion/, [Accessed on 2021-03-01].

Chowell, G., 2017. Fitting Dynamic Models to Epidemic Outbreaks with Quantified Uncertainty: A Primer for Parameter Uncertainty, Identifiability, and Forecasts. Infectious Disease Modelling 2, 3, 379-398.

Chowell, G., Hincapie-Palacio, D., Ospina, J., Pell, B., Tariq, A., Dahal, S., Moghadas, S., Smirnova, A., Simonsen, L., Viboud, C., 2016. Using Phenomenological Models to Characterize Transmissibility and Forecast Patterns and Final Burden of Zika Epidemics. PLoS Currents 8, 8.

Chowell, G., Tariq, A., Hyman, J., 2019. A novel sub-epidemic modeling framework for short-term forecasting epidemic waves. BMC Medicine 17, 164, 1-18.

Dal Molin Ribeiro, M.H., Gomes da Silva, R., Cocco Mariani, V., dos Santos Coelho, L., 2020. Short-term forecasting COVID19 cumulative confirmed cases: Perspectives for Brazil. Chaos, Solitons \& Fractals 135, 109853.

Gitto, S., Di Mauro, C., Ancarani, A., Mancuso, P., 2021. Forecasting national and regional level intensive care unit bed demand during COVID-19: The case of Italy. PLoS ONE 16, 2, e0247726. 
Goic, M., Bozanic-Leal, M.S., Badal, M., Basso, L.J., 2021. COVID-19: Short-term forecast of ICU beds in times of crisis. PLoS ONE 16, 1, e0245272.

Gupta, A., Sharda, R., 2013. Improving the science of healthcare delivery and informatics using modeling approaches. Decision Support Systems 55, 423-427.

Haman, J., 2017. Generalized Linear Models with ciTools, URL: https://cran.r-project.org/web/packages/ciTools/vignettes/ ciTools-glm-vignette.html, [Accessed on 2020-09-02].

Harvey, A.C., 1984. Time Series Forecasting Based on the Logistic Curve. The Journal of the Operational Research Society 35 , 7, 641-646.

Joshi, Y.V., Musalem, A., 2021. Lockdowns may lose up to half their impact on mobility after a month. URL: https://doi.org/ 10.21203/rs.3.rs-701885/v1, [Accessed on 2021-08-20].

Keeling, M.J., Danon, L., 2009. Mathematical modelling of infectious diseases. British Medical Bulletin 92, 1, 33-42. https: //academic.oup.com/bmb/article-pdf/92/1/33/954051/ldp038.pdf.

Ma, J., Dushoff, J., Bolker, B.M., Earn, D.J.D., 2014. Estimating Initial Epidemic Growth Rates. Bulletin of Mathematical Biology 76, 245-260.

Maier, B.F., Brockmann, D., 2020. Effective containment explains subexponential growth in recent confirmed COVID-19 cases in China. Science 368, 6492, 742-746.

Manca, D., Caldiroli, D., Storti, E., 2020. A simplified math approach to predict icu beds and mortality rate for hospital emergency planning under covid-19 pandemic. Computers Chemical Engineering 140, 106945.

Meo, S.A., Al-Khlaiwi, T., Usmani, A.M., Meo, A.S., Klonoff, D.C., Hoang, T.D., 2020. Biological and epidemiological trends in the Prevalence and Mortality due to Outbreaks of Novel Coronavirus COVID-19. Journal of King Saud UniversityScience 32, 2495-2499.

Moreau, V.H., 2020. Forecast predictions for the COVID-19 pandemic in Brazil by statistical modeling using the Weibull distribution for daily new cases and deaths. Brazilian Journal of Microbiology 51, 3, 1109-1115.

Nakagawa, K., Kanatani, T., 2021. Monday effect on confirmed cases of COVID-19 in Japan. medRxiv. https://www.medrxiv. org/content/early/2021/07/22/2021.07.20.21260858.full.pdf, doi: https://doi.org/10.1101/2021.07.20.21260858.

Papo, D., Righetti, M., Fadiga, L., Biscarini, F., Zanin, M., 2020. A minimal model of hospital patients' dynamics in covid-19. Chaos, Solitons \& Fractals 140, 110157.

Remuzzi, A., Remuzzi, G., 2020. COVID-19 and Italy: What Next? The Lancet 395, 1225-1228.

Richards, F.J., 1959. A Flexible Growth Function for Empirical Use. Journal of Experimental Botany 10, 2, $290-301$.

Roosa, K., Lee, Y., Luo, R., Kirpich, A., Rothenberg, R., Hyman, J., Yan, P., Chowell, G., 2020a. Short-term Forecasts of the COVID-19 Epidemic in Guangdong and Zhejiang, China: February 13-23, 2020. Journal of Clinical Medicine 9, 596, 1-9.

Roosa, K., Tariq, A., Yan, P., Hyman, J.M., Chowell, G., 2020b. Multi-model forecasts of the ongoing Ebola epidemic in the Democratic Republic of Congo, March - October 2019. Journal of the Royal Society Interface 17, 20200447, 1-12.

Tariq, A., Undurraga, E.A., Castillo Laborde, C., Vogt-Geisse, K., Luo, R., Rothenberg, R., Chowell, G., 2021. Transmission dynamics and control of COVID-19 in Chile, March-October, 2020. PLoS Neglected Tropical Diseases 15, 1, e0009070.

Torrealba-Rodríguez, O., Conde-Gutiérrez, R.A., Hernández-Javier, A.L., 2020. Modeling and prediction of COVID-19 in Mexico applying mathematical and computational models. Chaos, Solitons \& Fractals 138, 109946.

Vicuña, M.I., Vásquez, C., Quiroga, B.F., 2021. Forecasting the 2020 COVID-19 epidemic: A multivariate Quasi-Poisson regression to model the evolution of new cases in Chile. Frontiers in Public Health 9, 416-422.

Wang, X.S., Wu, J., Yang, Y., 2012. Richards model revisited: Validation by and application to infection dynamics. Journal of Theoretical Biology 313, 12-9.

Weibull, W., 1951. A Statistical Distribution Function of Wide Applicability. Journal of Applied Mechanics 18, 3, $293-297$.

Zreiq, R., Kamel, S., Boubaker, S., Al-Shammary, A.A., Algahtani, F.D., Alshammari, F., 2020. Generalized Richards model for predicting COVID-19 dynamics in Saudi Arabia based on particle swarm optimization Algorithm. AIMS Public Health 7, 4, 828-843. 